\title{
La comunicación periodística de la ciencia de la Universidad de Granada: el traductor como experto en localización
}

\author{
Ana Gregorio Cano \\ Universidad de Granada \\ anagregcan@ugr.es \\ Antonio MARÍN RUIZ \\ Universidad de Granada \\ amarin@ugr.es
}

Recibido: 30/07/2012

Aceptado: 22/10/2012

\begin{abstract}
Resumen
La divulgación de los avances científicos constituye hoy día una importante opción de empleo para periodistas y traductores de textos científico-técnicos. Dentro de este campo, la ciencia producida en las instituciones de enseñanza e investigación y, entre ellas, en las universidades, necesita de la labor de comunicadores especializados en divulgación de la ciencia y la tecnología y de traductores para alcanzar unos objetivos de difusión que vayan más allá del ámbito local y nacional para llegar a medios informativos de todo el mundo. En este sentido, el uso de las grandes agencias de divulgación de la ciencia y una labor traductora que haga un adecuado uso del concepto de localización son esenciales para alcanzar el éxito de las noticias científicas en el contexto internacional.

Palabras clave: periodismo científico, localización, competencia traductora, AlphaGalileo, EurekAlert!

\section{Communicating Science at the University of Granada... Journalism: the Translator as an Expert in Localization}

\begin{abstract}
The dissemination of scientific advances is currently an important employment site for journalist and scientific and technical translators. Science produced at educational and research institutions needs to be translated to achieve its goals: both dissemination new knowledge within the international scientific community to explore new research lines and informing society about the new scientific advances from which the general public will benefit to a greater or lesser extent. In this sense, scientific divulgation agencies and localization carried out by translators play a key role in the impact of scientific articles.

Keywords: Scientific Journalism, Localization, Translator Competence, AlphaGalileo, EurekAlert!

\section{Referencia normalizada}

GREGORIO CANO, Ana y MARÍN RUIZ, Antonio (2012): "La comunicación periodística de la ciencia de la Universidad de Granada: el traductor como experto en localización". Estudios sobre el mensaje periodístico. Vol. 18, núm. especial octubre, págs.: 465-474. Madrid, Servicio de Publicaciones de la Universidad Complutense.
\end{abstract}

Sumario: 1. Introducción; 1.1. La divulgación científica en la Universidad de Granada y la traducción: quid pro quo. 2. Metodología: el estudio de caso de la traducción español>inglés de noticias científicas de la UGR y su impacto en los medios de comunicación internacionales; 2.1. AlphaGalileo y EurekAlert!: dos repetidores indispensables; 2.2. Selección de casos, período de estudio y análisis de datos. 3. Exposición e interpretación de los resultados. 4. Conclusiones. 5. Referencias bibliográficas. 


\section{Introducción}

En este artículo abordaremos el caso de la traducción de noticias sobre la producción científica de la Universidad de Granada, realizadas por el Gabinete de Comunicación de dicha institución. En los últimos años, este Gabinete ha contado con la labor de periodistas científicos que han contado con la colaboración de expertos en traducción que han realizado las tareas de traducción y adaptación (localización) de noticias del idioma, cultura y público españoles a la lengua inglesa, orientándolas hacia una cultura científica y público internacionales. Las competencias profesionales de los traductores los hacen altamente versátiles para adaptar sus conocimientos y capacidades a diferentes campos de especialización y, más concretamente, a distintos lectores, lo que les permite obtener mejores resultados en la divulgación internacional de las noticias científicas. Tomaremos varios ejemplos de noticias que han alcanzado un gran impacto en medios digitales de índole internacional a través de la publicación de la traducción de las mismas en las plataformas internacionales de difusión de la ciencia AlphaGalileo y EurekAlert!

A través del análisis del impacto de estos ejemplos, podremos concluir que la colaboración entre periodistas científicos y traductores profesionales en el proceso de divulgación científica es clave tanto para abrir nuevos caminos y colaboraciones en el ámbito científico como para hacer llegar los nuevos avances científicos al público en general.

\subsection{La divulgación científica en la Universidad de Granada y la traducción: quid pro quo}

Los estudios de Traducción e Interpretación (TI) tienen en España un marcado enfoque práctico y multidisciplinar de las lenguas modernas. Tal y como apunta el libro blanco de Título de Grado en Traducción e Interpretación (ANECA, 2005), el plan de estudios de TI se encuentra altamente vinculado al mercado profesional siendo el grado de empleabilidad de sus titulados muy superior al de otros titulados por las diferentes competencias que desarrollan a lo largo del grado.

Una característica fundamental de los estudios de TI es que responden a las necesidades reales de un mercado laboral que exige perfiles multidisciplinares en los que el conocimiento y dominio de idiomas es cada vez más un punto clave y decisivo a la hora de conseguir un empleo. Los titulados en TI poseen competencias muy valoradas en el mercado de trabajo: capacidad de comunicación en más de una lengua tanto de forma oral como escrita, de trabajar en equipo, de cumplir plazos de entrega, de rendir bajo situaciones de presión temporal, de adquirir nuevos conocimientos y desarrollar nuevas habilidades en poco tiempo, etc.; así como la capacidad de trabajar con personas de diferentes lenguas y culturas fruto de la formación multilingüe y multicultural ofrecida durante sus estudios.

El traductor profesional aúna así una serie de conocimientos, habilidades y actitudes en dos lenguas y culturas que le convierten en candidato de muchos sectores profesionales entre los que se encuentra la divulgación científica en el caso en el que nos ocupa. 
Como ya hemos señalado, las características que reúnen los traductores (y/o intérpretes) los convierten, como ha sido el caso en el Gabinete de Comunicación de la UGR, en los mediadores ideales para la divulgación de los avances científicos. Una de las principales razones es su versatilidad, su capacidad de adaptación y su conocimiento, y en el mejor de los casos también alto dominio, de dos lenguas y culturas. En este caso, la importancia fundamental yace en el inglés, lengua que desde unos años a esta parte se ha convertido en la lingua franca, en el idioma de la comunicación internacional.

\section{Metodología: el estudio de caso de la traducción español>inglés de noticias científicas de la UGR y su impacto en los medios de comunicación interna- cionales}

Como mostraremos en la segunda parte de este artículo, el índice de impacto varía sustancialmente dependiendo de si la noticia ha sido circulada en español o en inglés, de ahí que la labor traductora y, por lo tanto, recurrir al inglés sea clave tanto para la difusión como para informar de los avances científicos que se están llevando a cabo en diferentes partes del mundo.

A continuación presentaremos varios ejemplos de noticias que han alcanzado un gran impacto en medios digitales de índole internacional a través de la publicación de la traducción de las mismas en las plataformas internacionales de difusión de la ciencia AlphaGalileo y EurekAlert!

\subsection{AlphaGalileo y EurekAlert!: dos repetidores indispensables}

El estudio de caso que presentamos en este artículo se fundamenta en la difusión de las noticias científicas producidas por la Universidad de Granada a través de dos plataformas o agencias internacionales de noticias: AlphaGalileo, impulsada, entre otros, por la Comisión Europea, y gestionada por la Fundación AlphaGalileo, y EurekAlert!, gestionada por la American Association for the Advancement of Science (AAAS). La experiencia ha demostrado que la publicación de la versión en inglés de las noticias científicas del Gabinete de Comunicación de la UGR confiere a la producción científica de la UGR un alcance mediático mucho más importante que la publicación de las mismas noticias en español a través de medios nacionales.

La colaboración del Gabinete de Comunicación de la UGR con estas dos plataformas comenzó en el año 2006. A partir de esta fecha, las noticias científicas publicadas por el Gabinete, y sus correspondientes traducciones en inglés y, más tarde, en francés, son incluidas en las bases de datos de ambas agencias virtuales. El método de trabajo es, en cierto modo, similar al que se utiliza con las noticias en español: todas las notas de prensa se publican en la página web del Gabinete de Comunicación dedicada a este fin (Noticias UGR - http://canalugres), que cuenta con diferentes secciones entre las que se encuentra "Noticias Ciencia UGR" y sus versiones en inglés (Science news UGR) y francés (Nouvelles de la Science UGR). Sin embargo, la difusión de las noticias no se realiza a través de estos soportes, sino que se lleva a cabo esencialmente a través de un directorio de medios locales, regionales y nacionales al que se les envía las noticias en español en la fecha de su publicación. El sistema de 
colaboración con AlphaGalileo y EurekAlert! permite el envío de las noticias traducidas a un gran número de medios internacionales, a modo de directorio de medios catalizado por ambas plataformas. ${ }^{1}$

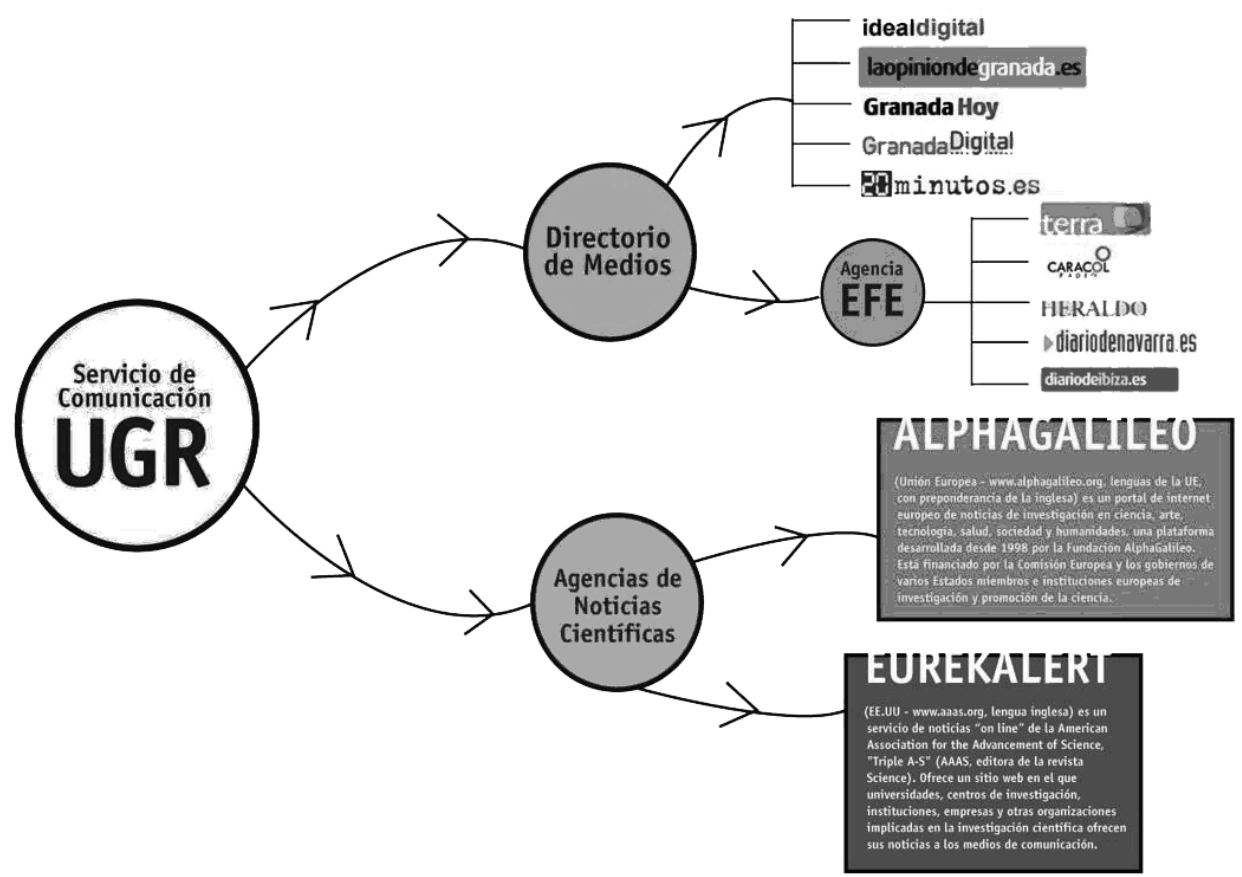

Figura 1. Sistema de difusión de noticias del Gabinete de Comunicación de la UGR (Centeno et al., 2007)

A pesar de que la estructura y las secciones de los dos sitios web no son del todo simétricas, el mecanismo de trabajo de estas plataformas internacionales de divulgación y popularización científica es similar. Ambas sirven de puente entre productores y receptores de la ciencia, es decir, AlphaGalileo y EurekAlert!, previa suscripción, ofrecen a las instituciones de investigación una plataforma en la que dar difusión pe-

1 Para los propósitos de este estudio, los autores reconocen una diferencia esencial entre la $p u$ blicación y la difusión de las noticias. Mientras que la publicación de las noticias, tanto en español como en inglés y francés, del Gabinete de Comunicación de la UGR se realiza en la página web de este servicio, la difusión de las mismas a los medios de comunicación se lleva a cabo gracias al envío de estas noticias directamente a los medios interesados. En el caso de las noticias en español, las noticias son difundidas a través del directorio de medios elaborado y constantemente actualizado por el propio Gabinete, que incluye medios y agencias en español, tanto locales como regionales y nacionales. Las noticias traducidas, por otro lado, son difundidas a través de las plataformas AlphaGalileo y EurekAlert!, que actúan como repetidores de las noticias y las envían a los medios y profesionales de la comunicación, así como a los miembros de la comunidad científica, que así lo han solicitado a través de una suscripción. 
riodística a su producción científica: se hacen eco de notas de prensa, reuniones científicas, novedades de la literatura científica publicada por universidades y otras instituciones de investigación, etc. y los ponen a disposición de profesionales de los medios de comunicación de todo el mundo, de otros investigadores y del público en general. Para ello, ofrecen al usuario motores de búsqueda con los que localizar cualquier artículo publicado en la página desde su creación, alertas de correo electrónico acerca de las nuevas noticias y otras novedades, alertas en formato RSS, etc., a las que el usuario puede acceder también a través de una suscripción.

\subsection{Selección de casos, período de estudio y análisis de datos}

Con el fin de ilustrar la influencia de la traducción al inglés de la producción científica de una institución de investigación sobre el impacto que la misma noticia, publicada en idiomas distintos, puede tener en los medios de comunicación, hemos seleccionado cinco noticias que han cubierto un ciclo largo de permanencia en los portales señalados y cuya traducción al inglés supuso un gran impulso en la visibilidad mediática de la investigación de la que informaban. Las cinco noticias escogidas obtuvieron un alto número de consultas en el registro que EurekAlert! elabora sobre el interés producido por las notas de prensa que publica en los profesionales de los medios de comunicación suscritos a su base de datos.

Una vez cotejadas las fechas de publicación en español (y consecuente envío a los medios en español del directorio de medios del Gabinete de Comunicación) y en inglés (y consecuente publicación en las plataformas AlphaGalileo y EurekAlert!), obtuvimos los impactos en medios de comunicación digitales de índole general que la publicación de sendas versiones lingüísticas alcanzó en el plazo de siete días, incluyendo el día de la publicación. La primera noticia seleccionada constituye una excepción a este criterio temporal de siete días, puesto que el período de publicación entre ambas versiones era de sólo cinco días y, para evitar solapamientos, recuperamos para la versión española el impacto en medios digitales de los cinco días previos a la publicación de la versión en inglés. Para la obtención de estos impactos, medidos en número de resultados obtenidos en medios de comunicación digitales, el Gabinete de Comunicación elabora diariamente, utilizando como herramienta el motor de búsqueda de noticias Google News, un Resumen de prensa de medios digitales, cuya base de datos puede ser libremente consultada para recuperar una determinada noticia sobre la UGR que apareció en los medios en un período de tiempo específico.

Todas las noticias, a diferentes niveles, alcanzaron mayor difusión en inglés que en español, con diferentes casos:

1. Amplia distancia temporal (meses) entre la difusión de la versión española a través del directorio de medios y la difusión de la versión inglesa a través de AlphaGalileo y EurekAlert!: noticias C, D y E.

2. Corta distancia temporal (días) entre la difusión de la versión española a través del directorio de medios y la difusión de la versión inglesa a través de AlphaGalileo y EurekAlert!: noticia A.

3. Difusión simultánea de las versiones española e inglesa, cada una a través de los correspondientes canales, ya mencionados: noticia B. 
Cada uno de estos casos proporciona diferentes matices al análisis de los resultados obtenidos a través de la recuperación de impactos en el Resumen de prensa de medios digitales, resultados que serán expuestos e interpretados en la siguiente sección.

\section{Exposición e interpretación de los resultados}

En la siguiente tabla se puede apreciar un resumen de los resultados obtenidos en la medición del impacto en medios de las cinco noticias seleccionadas.

\begin{tabular}{|c|c|c|c|c|}
\hline Noticia (es/en) & $\begin{array}{l}\text { Fecha } \\
\text { difusión } \\
\text { esp ariol } \\
\text { (directorio) }\end{array}$ & $\begin{array}{l}\text { Impacto en } \\
\text { medios* }\end{array}$ & $\begin{array}{l}\text { Fecha } \\
\text { difusión } \\
\text { inglés } \\
\text { (AG \& EA) }\end{array}$ & $\begin{array}{l}\text { Imp acto en } \\
\text { medios }\end{array}$ \\
\hline $\begin{array}{ccc}\text { A) Indicios fósiles } & y \\
\text { moleculares } & \text { ihsstrat } & \boldsymbol{b}\end{array}$ & & & & \\
\hline $\begin{array}{l}\text { historia de los grandes focos } \\
\text { de biodiversidad marina } \\
\text { Fossil and molecular } \\
\text { evidence reveals the history } \\
\text { of major marine biadiversity } \\
\text { hotspots }\end{array}$ & $02 / 08 / 2008$ & $\begin{array}{l}1 \text { es } \\
(02- \\
05 / 08 / 2008)\end{array}$ & $06 / 08 / 2008$ & $18 \mathrm{es}-7 \mathrm{en}$ \\
\hline $\begin{array}{l}\text { B) Un compuesto natual } \\
\text { extraido de la aceituna evita } \\
\text { el crecimiento de células } \\
\text { cancerigenas y previene su } \\
\text { aparición / A compound } \\
\text { extracted from olives inhibits } \\
\text { cancer cells growth and } \\
\text { prevents their appearance }\end{array}$ & $13 / 02 / 2008$ & 7 es & $13 / 02 / 2008$ & $19 \mathrm{en}$ \\
\hline $\begin{array}{l}\text { C) El I00\% de las personas } \\
\text { tiene en su organismo, al } \\
\text { menos, un tipo de pesticide, } \\
\text { que ingiere a traves del aire, } \\
\text { el agua o la comida / I0o\% of } \\
\text { people carry at least one type } \\
\text { of pesticide from the air, } \\
\text { water or food in their bodies }\end{array}$ & $29 / 11 / 2007$ & 1 es & 04/01/2008 & $0 \mathrm{es}-15 \mathrm{en}$ \\
\hline $\begin{array}{l}\text { D) Las amas de casa reciclan } \\
\text { más y tienen una mayor } \\
\text { conciencia ecológica que los } \\
\text { universitarios / Housewives } \\
\text { are more ecologically aware } \\
\text { and recycle more than } \\
\text { university students }\end{array}$ & $31,05 / 2007$ & 2 es & $27 / 12 / 2007$ & $4 e s-2 e n$ \\
\hline
\end{tabular}


E) Crean un sistema informático de visión nocturna en vehiculos que permite redisir el nímero de accidentes de tráfico / New night vision system reduces car accidents
$25 / 06 / 2007 \quad 4$ es $\quad 27 / 09 / 2007 \quad 0$ es -7 en

*Medido en términos de número de resultados obtenidos a través de una búsqueda con el motor Google News. Trabajamos con medios digitales. $\mathrm{AG}=$ AlphaGalileo; $\mathrm{EA}=\mathrm{Eu}-$ rekAlert!; es = español; en = inglés.

Figura 2. Impacto en medios digitales de las noticias científicas de la UGR en español y de su traducción en inglés.

La primera columna consta de los titulares de las noticias, en sus versiones española e inglesa, tal como fueron publicadas ${ }^{2}$. Las columnas segunda y cuarta indican las fechas de difusión, de la versión española a través del directorio de medios del Gabinete de Comunicación de la Universidad de Granada y de la versión inglesa a través de las páginas web de AlphaGalileo y EurekAlert!. En la tercera y quinta columna se pude comprobar el número de impactos, tanto en español como en inglés, que la difusión de las noticias alcanzó en un período de siete días, incluyendo la fecha de difusión. El primer resultado destacable que se observa en este análisis es, sin duda alguna, que a excepción de la noticia $\mathrm{B}$, difundida simultáneamente en ambos idiomas, la publicación en español no genera resultados en la medición de impactos en medios en lengua inglesa.

Al proceder a un análisis individual de las distintas noticias se comprueba que la noticia A, con un período de cinco días entre su difusión en español y su difusión en inglés, alcanza tan sólo un impacto en medios digitales, mientras que su difusión en inglés no solamente alcanza un número mayor de impactos (7), sino que eleva hasta 18 el número de impactos en español que la noticia alcanza tras su difusión a través de AlphaGalileo y EurekAlert!

La noticia B fue difundida simultáneamente en español y en inglés y, una vez más, el número de impactos en medios digitales es mucho mayor (más del doble) en inglés (19) que en español (7).

Las versiones en español y en inglés de las noticias C, D y E fueron difundidas con varios meses de diferencia. Los casos $\mathrm{C}$ y E muestran resultados similares, con un número mayor de impactos de la versión inglesa. En el caso D, la difusión de las noticias en inglés no sólo alcanzó un impacto similar a la versión española, sino que repercutió en la nueva aparición de esta noticia en medios en español, como El País o El Mundo, que en un primer no se habían hecho eco de la noticia. Es cierto que, según los resultados, el impacto de la difusión en inglés de la noticia D (diciembre de 2007) su-

${ }^{2}$ Los enlaces correspondientes al texto completo de las noticias se reseñan en las referencias bibliográficas. 
puso un impacto similar al de su difusión en español (mayo de 2007); no obstante, nos gustaría señalar que, a la luz de los resultados generales de este estudio y de nuestra experiencia en comunicación de la ciencia y traducción, estamos convencidos de que si la difusión en inglés de la noticia $\mathrm{D}$ se hubiese producido en una fecha distinta a finales de diciembre, su impacto en medios de ambos idiomas habría sido mucho mayor.

Si se tienen en cuenta estos resultados, se puede apreciar claramente el diferente impacto de la publicación de la misma noticia en diferentes idiomas y utilizando plataformas (repetidores) distintas. No solamente se comprueba que la noticia alcanza un mayor número de medios en inglés que en español, sino que la publicación en inglés repercute en una mayor difusión en español, hipótesis que se confirma en las noticias A, B y D. En el caso B, a pesar de que la difusión de ambas versiones lingüísticas es simultánea, estamos convencidos de que la difusión de la noticia en inglés en medios internacionales influye de forma decisiva en el alto número de impactos que alcanza la noticia en español, puesto que nuestra experiencia confirma que los medios españoles toman la noticia de la versión inglesa y acceden a la versión española a través de la primera. No obstante, somos conscientes de la dificultad en la demostración empírica de esta influencia retroactiva, si no es en base a una encuesta de hábitos de los medios de comunicación implicados. Asimismo, consideramos que un análisis temporal más amplio y con una muestra considerablemente mayor arrojaría más luz sobre esta cuestión.

\section{Conclusiones}

El presente estudio ha puesto de manifiesto la importancia de la colaboración entre periodistas científicos universitarios y traductores en la difusión de los avances científicos, tanto hacia la comunidad científica en general como hacia la sociedad, la gran beneficiaria y destinataria final de dichos avances.

Las competencias profesionales de los traductores, que hemos destacado en la primera parte, los hacen una pieza clave en el proceso de adaptación de las noticias publicadas en español a un lector y una cultura científica internacionales, lo que mejora considerablemente los resultados de difusión de los nuevos avances a escala internacional. Además, la divulgación de las noticias científicas a través de traducciones de calidad, que utilidad una terminología correcta y están adaptadas al lector de cada caso en particular, nos asegura la fiabilidad como fuente de información y multiplica la velocidad de difusión y de repetición de las noticias en los medios internacionales.

La publicación de la noticia en inglés en medios internacionales repercute en su impacto en medios escritos en español, por lo que podemos afirmar que la vía de transmisión del conocimiento científico y tecnológico en la actualidad es el inglés. De este modo, consideramos que sería muy útil no quedarnos en un análisis del impacto del uso (o tal vez, abuso) del inglés como vehículo científico, sino que sería interesante que posteriores trabajos arrojasen luz sobre las implicaciones tanto lingüísticas como sociológicas e, incluso, éticas de este fenómeno de Englishisation o utilización del inglés como lingua franca.

La labor del traductor, en cualquier caso, se revela una vez más indispensable en la transmisión del conocimiento y la modulación del idioma, a través de la creación 
de neologismos o la utilización de préstamos e, incluso, calcos con el fin de incorporar las novedades científico-técnicas al conocimiento social y, de este modo, a la popularización de los avances científicos.

\section{Referencias bibliográficas}

AGENCIA NACIONAL DE EVALUACIÓN DE LACALIDAD YACREDITACIÓN (ANECA) (2004): Libro blanco del título de grado en Traducción e Interpretación.. Disponible en: http://www.aneca.es/var/media/150288/libroblanco_traduc_def.pdf

ALPHAGALILEO.ORG - the Internet-based News Centre for European Science, Engineering and Technology, http://www.alphagalileo.org

CENTENO CUADROS, Carlos; ORTEGA GARRIDO, María del Mar; GALIANO SÁNCHEZ, Carmen; y SÁNCHEZ RUIZ, Laura (2007): "La comunicación de la ciencia y el papel de las agencias de noticias: el caso de la melatonina", póster presentado en el IV Congreso sobre Comunicación social de la ciencia, Consejo Superior de Investigaciones Científicas (CSIC), Madrid,

EUREKALERT! - Science News, http://www.EurekAlert.org

GABINETE DE COMUNICACIÓN DE LA UNIVERSIDAD DE GRANADA:

31 de mayo de 2007: Las amas de casa reciclan más y tienen una mayor conciencia ecológica que los universitarios, http://prensa.ugr.es/prensa/investigacion/verNota/prensa.php?nota $=3839$

$\sim 18$ de junio de 2007: Housewives are more ecologically aware and recycle more than university students, http://prensa.ugr.es/prensa/research/verNota /prensa.php?nota $=463$

$\sim 25$ de junio de 2007: Crean un sistema informático de visión nocturna en vehículos que permite reducir el número de accidentes de tráfico, http://prensa.ugr.es/prensa/investigacion/verNota/prensa.php?nota=3884

$\sim 29$ de junio de 2007: New night vision system reduces car accidents, http://prensa.ugr.es/prensa/research/verNota/prensa.php?nota $=467$

$\sim 29$ de noviembre de 2007: 100\% of people carry at least one type of pesticide from the air, water or food in their bodies, http://prensa.ugr.es/prensa/research/verNota/prensa.php?nota $=498$

$\sim 29$ de noviembre de 2007: El 100\% de las personas tiene en su organismo, al menos, un tipo de pesticida, que ingiere a través del aire, el agua o la comida, http://prensa.ugr.es/prensa/investigacion/verNota/prensa.php?nota $=4161$

$\sim 13$ de febrero de 2008: A compound extracted from olives inhibits cancer cells growth and prevents their appearance, $\mathrm{http} / /$ prensa.ugr.es/prensa/research/verNota/prensa.php?nota $=513$

$\sim 13$ de febrero de 2008: Un compuesto natural extraido de la aceituna evita el crecimiento de células cancerígenas y previene su aparición, http://prensa.ugr.es/prensa/investigacion/verNota/prensa.php?nota $=4276$ 
$\sim 2$ de agosto de 2008: Fossil and molecular evidence reveals the history of major marine biodiversity hotspots, http://prensa.ugr.es/prensa/research/verNota /prensa.php?nota $=553$

$\sim 2$ de agosto de 2008: Indicios fósiles y moleculares ilustran la historia de los grandes focos de biodiversidad marina, http://prensa.ugr.es/prensa/investigacion/verNota/prensa.php?nota $=4680$

Noticias Ciencia Universidad de Granada, http://prensa.ugr.es/prensa/investigacion/index.php

RESUMEN DE PRENSA DE MEDIOS DIGITALES - Gabinete de Comunicación de la Universidad de Granada, http://prensa.ugr.es/prensa/campus/dossierdigital.php

\section{Ana GREGORIO CANO}

anagregcan@ugr.es

Universidad de Granada

Profesora de Traducción e Interpretación. Universidad de Granada. Departamento de Traducción e Interpretación. Calle Buensuceso, 11. Granada, C.P. 18002.

\section{Antonio MARÍN RUIZ}

amarin@ugr.es

Universidad de Granada

Responsable de gestión de proyectos y difusión del Centro de Enseñanzas Virtuales de la

Universidad de Granada (CEVUG). C/Real de Cartuja, nº 36-38. Granada, C.P. 18071. 